\title{
Evaluation of Temporomandibular Joint Dis- orders and Related Factors in Patients Refer- ring to Dental School of Isfahan Islamic Azad University in 2019
}

\author{
M Falahati ${ }^{1}$, F Golmohammadi *@ 2 , R Darabi ${ }^{2}$, M Jafari ${ }^{3}$ \\ 1-Dentistry Graduate Student, School of Dentistry, Isfahan (Khorasgan) Branch, Islamic Azad University, Isfa- \\ han, Iran \\ 2- Assistant professor, Department Of Prosthetic Dentistry, Faculty of Dentistry, Islamic Azad University, Isfah- \\ an, Iran \\ 3- Postgraduate Student, Department Of Prosthetic Dentistry, Faculty of Dentistry, Islamic Azad University, \\ Isfahan, Iran
}

\begin{tabular}{l}
\hline ARTICLE INFO \\
\hline Article History \\
Received: Apr 2020 \\
Accepted:May 2020 \\
ePublished: Jun 2020 \\
\hline Corresponding author: \\
F Golmohammadi, \\
Assistant professor, \\
Department Of Prosthet- \\
ic Dentistry, Faculty of \\
Dentistry, Islamic Azad \\
University, Isfahan, Iran \\
Email: Farnoosh.gol@ \\
gmail.com
\end{tabular}

Introduction:

The term"temporomandibular disorders" (TMD) encompasses the clinical complications of the masticatory musculature and the temporomandibular joint (TMJ). ${ }^{(1-3)}$ TMD has a multifactorial etiology, including inflammation and swelling of the joints, trauma, stress, some dental appliances, bruxism and clenching, iatrogenic injuries related to surgery and radiotherapy,

\begin{abstract}
Background and Aim: Temporomandibular disorders (TMD) encompass several entities with different etiologies. Considering the high prevalence of TMD and the effect of this complication on the development of orofacial complications, this study aimed to determine the prevalence of TMD and its associated factors in patients referring to the Department of Prosthodontics of the Dental School of Isfahan Islamic Azad University, Isfahan, Iran.

Materials and Methods: IA cross-sectional study was conducted on 202 patients for TMD evaluation. Standardized checklists and paraclinical measures were used. SPSS was used with chi-square test, t-test, and one-way analysis of variance (ANOVA) to perform the statistical analysis with the level of significance set at $\mathrm{P}<0.05$.

Result: The prevalence of TMD in 202 patients was $43.6 \%$. The average age of TMD patients was significantly higher than that of people without TMD ( $\mathrm{P}=0.012)$. There was a significant difference between the two groups (with and without TMD) in the history of maxillofacial trauma $(\mathrm{P}=0.014)$ and restricted mouth opening $(\mathrm{P}=0.02)$. The Dawson classification, maxillofacial surgery, orthodontic treatment $(\mathrm{P}=0.42)$, and parafunctional habits $(\mathrm{P}=0.46)$ were not significantly different between the two groups. Conclusion:Since the frequency of TMD is remarkably high, dentists should look for signs of TMD on a routine schedule to minimize the long-term effects of this disorder. There was no significant relationship between TMD and the related factors, except for the trauma. It is recommended to study the etiology of this disease considering its recognized complications.
\end{abstract}

Keywords: Temporomandibular Disorders, Dental Occlusion, Occlusal, Risk factors Res Dent Maxillofac Sci 2020;5(3):21-29

as well as some genetic disorders, such as hemifacial microsomia, hemifacial atrophy, juvenile rheumatoid arthritis, and mandibular shift and severe overbite. ${ }^{(1-3)}$

It has been suggested that the disorder is 1.5-2 times more prevalent in women; $80 \%$ of patients being treated for TMD are women. 
There may be a correlation between the pathogenesis of TMD and the female hormonal axis considering the gender and age distribution of TMD. ${ }^{(4)}$

The pain can be mild to severe and chronic or temporary. The statistics issued by some studies indicate that this disease affects an average of 30$40 \%$ of people in each community. ${ }^{(5)}$

Okeson introduces the most common TMD as muscular disorders, which are characterized by two important signs of pain and disability as well as headache and dysfunction that appears as decreased mandibular movement and is a prevalent finding in masticatory muscle disorders. ${ }^{(6)}$

The signs and symptoms related to TMJ disturbances include the inability to chew foods, facial pain, sensitivity and jaw pain, ear pain, clicks or sharp sense during mouth opening or chewing, jaw locking that makes mouth opening and closing difficult, headache, and painful and uneven chewing due to premature contacts. ${ }^{(7)}$ The absence of pain or jaw movement limitations correlated with clicking may rule out TMD. ${ }^{(8)}$

Common TMD-associated comorbidities include arthralgia, myalgia, local myalgia, myofascial pain, referring myofascial pain, disk displacement disorders, degenerative joint disease, subluxation, and headache. ${ }^{(9)}$

Numerous epidemiological studies have reported different prevalence rates for TMJ problems in children and adults around the world due to different clinical methods. One in 6 children and adolescents have clinical signs of TMJ disorders. The most prevalent sign is clicking. ${ }^{(10)}$ Some studies show that females have TMD signs and symptoms more frequently than males. TMJ problems are four times more frequent in women and they need treatment for these problems three times more than men do. ${ }^{(11)}$

TMD is the most common complaint of dental patients after toothache; around $40-60 \%$ of the population exhibit at least one of the signs and symptoms of TMD..$^{(1,2)}$

Although many complications can be prevented by being aware of TMD prevalence and associated factors, some studies refute these hypotheses, stating that there is no scientific evidence supporting such a relationship. Hence, we decided to study the patients referring to the Dental School of Isfahan Islamic Azad University,
Isfahan, Iran, using standard checklists and paraclinical measures. TMD and its prevalence were determined and related risk factors were investigated. TMD is the most common complaint of dental patients after toothache; around $40-60 \%$ of the population exhibit at least one of the signs and symptoms of TMD. ${ }^{(1,2)}$

Although many complications can be prevented by being aware of TMD prevalence and associated factors, some studies refute these hypotheses, stating that there is no scientific evidence supporting such a relationship. Hence, we decided to study the patients referring to the Dental School of Isfahan Islamic Azad University, Isfahan, Iran, using standard checklists and paraclinical measures. TMD and its prevalence were determined and related risk factors were investigated.

\section{Materials and Methods:}

This descriptive cross-sectional study investigated the prevalence of TMD among patients referring to the Department of Prosthodontics of the Dental School of Isfahan Islamic Azad University, Isfahan, Iran. The ethical code of the study was received from the Research Ethics Committee of Isfahan Islamic Azad University (IR.IAU.KHUISF.REC.1398.022). The diagnostic criteria for temporomandibular disorders (DC/ TMD) were used for the assessments. ${ }^{(9)}$

To conduct the research, two questionnaires, a ruler, a mirror, and a stethoscope (the auxiliary tool for examination) were prepared. All selected subjects who met the criteria were informed about the details of the study and were requested to sign an informed consent prior to the study. The first questionnaire included questions regarding joint sounds, joint pain, jaw opening limitation, a history of trauma, parafunctional habits, a history of orthodontic treatment, therapeutic medications, and systemic disease.

In the second questionnaire, the parts of the first questionnaire were tested with a clinical examination. In the third part of the form, jaw movements were investigated. To record the maximum mouth opening, we asked patients to open their mouths as much as they could. Then, we measured the distance between the edge of 
the maxillary central incisor and the edge of the mandibular central incisor plus overbite as the maximum opening without any force. Then, we asked patients to open their mouths to the maximum even if it causes pain. By placing the thumb on the upper central incisor and the index finger of the same hand on the lower incisor, moderate pressure was applied to open the mouth, measuring maximum jaw opening with force ${ }^{(12,13)}$ For measuring the lateral movements, the examiner marked the lower incisor and asked the patient to move the jaw to the left and right. The distance from the upper midline to the recorded point on the lower jaw is representative of the amount of lateral movement to the right and left. We considered jaw movement limitations when mouth opening without help and pain reached to less than $40 \mathrm{~mm}$ and maximum opening with help increased up to $5 \mathrm{~mm}$ or when the lateral movements were less than $8 \mathrm{~mm} .^{(12,13)}$

The fourth part of the questionnaire included the mouth opening pattern, which was recorded as direct, deviation, deflection, and variable. The fifth section included the clinical examination of the TMJs and the posterior connections. Pain on each side was also recorded.

Next, the masticatory muscles were palpated. The pressure was started from zero and was increased within 10 seconds until the fingernails became pale. The examination involved the temporal muscle in three areas, including the upper frontal lobe and the anterior part of the TMJ, the middle lobe on the TMJ and the maxillary arch, and the posterior region of the muscle, superior and posterior to the ear. At least one trigger point of pain must be in the region of palpation. If the examiners were not confident about the correct positioning of the fingers, they asked the patient to clench to be able to touch the muscle more easily. During the palpation, we asked the patient about pain and discomfort. The origin and insertion of the masseter muscle were touched bilaterally.

Due to discomfort upon palpation of the medial pterygoid muscle and the lateral wall of the pharynx, as well as difficulty in the palpation of the lateral pterygoid muscle, the functional manipulation technique was used to assess the symptoms related to the medial pterygoid muscle and the upper and lower bounds of the lateral pterygoid muscle. ${ }^{(12)}$

In the seventh section, edentulous areas were observed and recorded. Posterior edentulous ridges in each quadrant excluded patients from the study because the extraction of posterior teeth disrupts the occlusion and might change the anterior guidance.

The eighth part comprised occlusion classification according to the Dawson classification as follows:

Class I: There is no discomfort in the TMJ region even when firmly loaded, and the maximum intercuspation (MIC) is in coordination with the centric relation $(\mathrm{CR})$.

Class IA: Adaptive deformation of the intracapsular structures. The MIC is in harmony with the CR. Since it is not possible to examine the intracapsular structures, in this study, both Class IA and I were considered as Class I.

Class II: To reach the MIC, the condyles must displace from a verifiable CR. There is also a Class IIA, which says that to reach the MIC, the condyles must displace from an adapted CR. Since these two were not distinguishable by examination, both were considered as Class II.

Class III: The CR is not reliable, and the TMJs cannot tolerate loading without tenderness or tension; therefore, the MIC cannot be determined until the TMJ problem is resolved. This condition has the potential to return to normal, but during the examination, the patient is not capable of enduring pressure without discomfort.

Class IV: The occlusal relationship is in an active stage of progressive disorder because of pathologically unstable TMJs.

The last section of this questionnaire studied parafunctional habits. While we asked in the first form about bruxism, we did the clinical examination to determine the presence of bruxism that patients are unaware of. As it was found in a previous study, the prevalence of bruxism in the survey was $8-12 \%$ but when the clinical oral observation was carried out, the statistics reached to $40-80 \%$. ${ }^{(3)}$

According to previous studies, $20-50 \%$ of patients have bruxism without being aware of it. ${ }^{(4)}$ The most common symptoms of night bruxism are fatigue of the masticatory muscles and the jaw, facial pain, and tension headaches in the morning. ${ }^{(4)}$ Some problems, such as dental wear, 
broken dental restorations, pain under removable dentures, scalloped tongue, loosened and sensitive teeth, and hypertrophy of the masticatory muscles, especially in the masseter muscle and sometimes in the temporalis muscle, could be signs of increased jaw activity and severe bruxism in long term. ${ }^{(12,13)}$ Dental wear manifested during eccentric movements is due to parafunctional habits. Oral habits, chemicals, and other factors may be present if worn surfaces cannot match the contacts properly. ${ }^{(12)}$

SPSS (SPSS Inc., Chicago, IL, USA) was used with chi-square test, t-test, and one-way analysis of variance (ANOVA) to perform the statistical analysis with the level of significance set at $\mathrm{P}<0.05$.active stage of progressive disorder because of pathologically unstable TMJs.

The last section of this questionnaire studied parafunctional habits. While we asked in the first form about bruxism, we did the clinical examination to determine the presence of bruxism that patients are unaware of. As it was found in a previous study, the prevalence of bruxism in the survey was $8-12 \%$ but when the clinical oral observation was carried out, the statistics reached to $40-80 \%$. ${ }^{(3)}$

According to previous studies, $20-50 \%$ of patients have bruxism without being aware of it. ${ }^{(4)}$ The most common symptoms of night bruxism are fatigue of the masticatory muscles and the jaw, facial pain, and tension headaches in the morning. ${ }^{(4)}$ Some problems, such as dental wear, broken dental restorations, pain under removable dentures, scalloped tongue, loosened and sensitive teeth, and hypertrophy of the masticatory muscles, especially in the masseter muscle and sometimes in the temporalis muscle, could be signs of increased jaw activity and severe bruxism in long term. ${ }^{(12,13)}$ Dental wear manifested during eccentric movements is due to parafunctional habits. Oral habits, chemicals, and other factors may be present if worn surfaces cannot match the contacts properly. ${ }^{(12)}$

SPSS (SPSS Inc., Chicago, IL, USA) was used with chi-square test, t-test, and one-way analysis of variance (ANOVA) to perform the statistical analysis with the level of significance set at $\mathrm{P}<0.05$.
Results:

Data were collected randomly from among 202 patients (101 females and 101 males; mean age of 33.8 years).

The results of the anamnestic questionnaire revealed that 88 subjects $(43 \%)$ were diagnosed with TMD. Among the factors affecting the prevalence of this condition, history of maxillofacial trauma $(10.9 \%)$, surgery (14.4\%), and orthodontic treatment $(9.9 \%)$ were the most prevalent.

The average age of patients with and without TMD was $35.2 \pm 7.1$ and $32.7 \pm 6.7$ years old, respectively. According to the independent t-test, the average age of TMD patients was significantly higher than that of subjects without TMD $(\mathrm{P}=0.012)$. The frequency of TMD was $52.5 \%$ among men and $47.5 \%$ among women with no statistically significant differences $(\mathrm{P}=0.26)$.

The chi-square test showed that the prevalence of TMD was significantly higher in subjects with a history of maxillofacial trauma $(\mathrm{P}=0.014)$ but there was no significant relationship between the prevalence of TMD and a history of surgery $(\mathrm{P}=0.08)$ or orthodontic treatment.

The independent $t$-test indicated that the mean mouth opening range $(\mathrm{P}=0.02)$ and the amount of movement to the right $(\mathrm{P}=0.011)$ were significantly less in TMD patients but the average overbite was not significantly different between subjects with and without TMD $(\mathrm{P}=0.024)$. On the other hand, the mean range of movement to the left side was not significantly different between the two groups $(\mathrm{P}=0.56)$.

Table 1 represents the frequency distribution of pain in different areas in subjects with and without TMD. Pain in wide mouth opening, in the lateral attachments of right and left TMJs, in the right and left posterior attachments of the TMJ, and in the masseter muscle showed a significant difference between the two groups with and without TMD. Pain in the temporalis muscle did not show any significant difference between the two groups with and without TMD.

The study of pain in the pterygoid muscles showed that none of the patients in this study had pain in the upper and lower bounds of the pterygoid muscles but three patients had pain in the medial pterygoid muscle (two of them had TMD). 
Table 1- Distribution of pain in different areas

\begin{tabular}{lccccc}
\hline & \multicolumn{1}{c}{ Without TMD } & \multicolumn{2}{c}{ With TMD } & \\
\hline & Number & Percentage & Number & Percentage & P-Value \\
\hline $\begin{array}{l}\text { Pain in wide } \\
\text { mouth opening }\end{array}$ & 7 & 6.4 & 19 & 22.4 & 0.001 \\
\hline $\begin{array}{l}\text { Pain in lateral } \\
\text { part of right } \\
\text { TMJ }\end{array}$ & 4 & 3.5 & 16 & 21.8 & 0.001 \\
\hline $\begin{array}{l}\text { Pain in lateral } \\
\text { part of left TMJ }\end{array}$ & 1 & 0.9 & 11 & 12.5 & 0.001 \\
\hline $\begin{array}{l}\text { Pain in posterior } \\
\text { attachments of } \\
\text { right TMJ }\end{array}$ & 4 & 3.5 & 13 & 14.8 & 0.004 \\
\hline $\begin{array}{l}\text { Pain in posterior } \\
\text { attachrments of } \\
\text { left TMJ }\end{array}$ & 1 & 0.9 & 10 & 11.4 & 0.001 \\
\hline $\begin{array}{l}\text { Pain in right } \\
\text { temporal muscle }\end{array}$ & 0 & 0 & 2 & 2.3 & 0.11 \\
\hline $\begin{array}{l}\text { Pain in left } \\
\text { temporal muscle }\end{array}$ & 0 & 0 & 0 & 0 & 1 \\
\hline $\begin{array}{l}\text { Pain in right } \\
\text { masseter muscle }\end{array}$ & 2 & 1.8 & 14 & 15.9 & $0.001>$ \\
\hline $\begin{array}{l}\text { Pain in left } \\
\text { masseter muscle }\end{array}$ & 1 & 0.9 & 6 & 6.8 & 0.022 \\
\hline
\end{tabular}

$\mathrm{TMD}=$ Temporomandibular

Disorders, TMJ=Temporomandibular Joint

According to the chi-square test, there was no significant relationship between TMD and occlusion status based on the Dawson classification $(\mathrm{P}=0.82)$. The chi-square test showed no significant correlation between TMD and occlusion status, according to the Angel classification, or between TMD and parafunctional habits $(\mathrm{P}=0.19)$. According to the results of our study, among the factors affecting TMD, age, history of maxillofacial trauma, and history of orofacial surgery had a significant effect on TMD but a history of orthodontic treatment had no significant effect on TMD. Also, the results of our study showed that 26 patients $(25.2 \%)$ with muscular pain had a history of surgery in the mandibular region. There were also 60 patients with pain upon TMJ palpation; $25.2 \%$ of them had a history of maxillofacial surgery. Also, 118 patients (51\%) had joint sounds (clicks); of them, eight patients $(6.8 \%)$ had a history of maxillofacial surgery. In addition, 26 patients $(12.9 \%)$ had pain in wide mouth opening; of them, seven patients $(26.9 \%)$ had a record of maxillofacial surgery.

In this study, 20 patients had a history of orthodontic treatment; 13 of them $(11.4 \%)$ did not have TMD, and seven of them (8\%) did. None of the patients with TMJ pain had a history of orthodontic treatment. Nine patients $(45 \%)$ exhibited joint sounds; of them, two patients (11.1\%) experienced pain in wide mouth opening.

Of the 202 patients, $28.7 \%$ were in Class I Dawson and $71.3 \%$ were in Class II Dawson. $55.2 \%$ of Class I Dawson patients and 67 patients from Class II Dawson (46.5\%) experienced masticatory muscle pain. $23.3 \%$ of patients had TMD, including 28 patients in Class I Dawson (23.3\%) and 19 patients in Class II Dawson $(76.7 \%)$.

Furthermore, 135 subjects $(66.8 \%)$ exhibited joint sounds; of them, $36.3 \%$ were in Class I Dawson and $63.7 \%$ were in Class II Dawson.

Among 26 patients with pain in wide mouth opening, eight patients $(30.8 \%)$ were in Class I Dawson and 18 patients $(69.2 \%)$ were in Class II Dawson.

In addition, 39 male patients (38.6\%) and 58 women $(57.4 \%)$ had masticatory muscle pain. Twenty-six men (25.7\%) and 33 women $(32.7 \%)$ had pain upon TMJ palpation. Fifty-six women (55.4\%) and 79 men (78.2\%) exhibited joint sounds. Fifteen men (14.9\%) and 11 women $(10.9 \%)$ experienced pain in wide mouth opening.

\section{Discussion:}

From the 202 patients that participated in this study, $43.6 \%$ had TMD; therefore, TMD can be considered as a prevalent disease with orofacial features.

In a study by Kamisaka et al, the prevalence of TMD was around $18 \% .^{(14)}$ In that study, cases were selected from among normal people, not symptomatic patients. ${ }^{(14)}$ In a study conducted by Lasemi et al, the prevalence of TMD in a group of symptomatic patients was $34.1 \%{ }^{(15)}$ In a study by Fardi, the prevalence of TMD in a group of students was $5.2 \%{ }^{(16)}$ In another study conducted by Ebrahimi et al on a group of adults, the prevalence of TMD was reported to be $75 \% .{ }^{(7)}$ Perhaps, the most important reason for the difference in the aforementioned studies is related to the study of TMD problems as well as the target populations of different studies and perceptions of patients in the expression of pain.

In our study, the patients that referred to the dental clinic were studied and clinically examined. A high prevalence of TMD among these pa- 
tients is normal whereas, in previous studies, including the studies by Kamisaka et al ${ }^{(14)}$, Lasemi et al ${ }^{(15)}$, Fardi ${ }^{(16)}$, and Ebrahimi Saravi et al ${ }^{(7)}$,

The statistical community had been selected from among different social groups and subjects with no clinical symptoms. However, regardless of the target population, the prevalence of stimulating factors in different populations may depend on different factors, such as individual characteristics, diet, traffic accidents, etc. According to numerous studies, Iran is one of the countries with a high number of traffic accidents; therefore, the rate of trauma to the orofacial muscles and the TMJ could be higher.

In this study, three factors, including maxillofacial trauma, history of surgery, and orthodontic treatment were present in at least $34 \%$ of patients, which is a high percentage.

In other studies, different factors have been studied from different viewpoints. In the study by Kamisaka et al, social factors exhibited a significant correlation with TMD. ${ }^{(14)}$ In the study by Lasemi et al, age, gender, parafunctional habits, trauma, orthodontic treatment, posterior edentulous ridge, depression, and anxiety showed a significant correlation with TMD. ${ }^{(15)}$ In the study by Fardi, bruxism, trauma, gender, and occlusion were effective in the incidence of TMD. ${ }^{(16)}$ In a study by Rokaya et al, most TMD patients had a history of head trauma, psychological stress, dental treatment, or dental problems. ${ }^{(17)}$ Staniszewski et al showed that physiological factors, such as abnormal secretion of cortisol hormone, are effective in the incidence of TMD. ${ }^{(5)}$

Our results showed that, among the factors affecting TMD, age and maxillofacial trauma have a significant effect on TMD but a history of orthodontic treatment and maxillofacial surgery did not have a significant effect on TMD. According to our results, the prevalence of some TMD symptoms was high in patients with a history of maxillofacial surgery. From 103 patients (51\%) with muscular pain, 26 patients $(25.2 \%)$ had a history of jaw surgery. There were also 60 patients with pain upon TMJ palpation; of them, $25.2 \%$ had a record of maxillofacial surgery. According to the results of our study, 118 patients (51\%) exhibited joint sounds; eight of them $(6.8 \%)$ had a history of maxillofacial surgery. Twenty-six patients (12.9\%) experienced pain in wide mouth opening; of them, $26.9 \%$ had a history of maxillofacial surgery.

Kamisaka et al stated that a history of trauma and gender had a significant effect on the incidence of TMD. ${ }^{(14)}$ In the study by Lasemi et al, parafunctional habits, trauma, a history of orthodontic treatment, posterior edentulous region, anxiety, and depression were more prevalent in TMD patients. ${ }^{(15)}$ In the study by Fardi, bruxism, trauma, gender, and type of occlusion showed a significant effect on TMD. (16)

In the study by Rokaya et al, a history of head trauma, psychological stress, dental treatment, or dental problems had a higher frequency in TMD patients. ${ }^{(17)}$ Therefore, it can be concluded that among the various factors affecting the incidence of TMD, demographic features, such as high age and being female, as well as a history of trauma, have a significant effect on TMD.

The results of our study showed that the prevalence of pain in some cases is significantly different in patients with and without TMD. The pain was more severe in wide mouth opening and the posterior joint attachments in patients with TMD.

The results of several studies have shown a relationship between pain in the temporomandibular region and TMD; therefore, in the standard checklists, restricted mouth opening and pain in various parts of the TMJ are the initial and most common symptoms of TMD. In this regard, the results of studies conducted by Baghaee et al, (2), Sahebi and Bostani Amlashi, (3) Lotfikamran et al, (4) and Heydari et al ${ }^{(18)}$ indicate that pain in different parts of the TMJ combined with restricted mouth opening and occlusal disorders are the most common symptoms in patients with TMD.

Although the Angel classification does not consider a detailed position and status of the condyles, this classification has been used routinely to show the relationship of the mandibular arch to the maxillary arch in several studies. On the other hand, analysis of any occlusion requires careful inspection of the maximal intercuspation position concerning both the position and the condition of the TMJs.

What might appear to be an ideal Angel Class I may be an Angel Class II with extreme overjet if the joint position is not considered after condyles place in the CR. In terms of the stability and 
comfort of the masticatory muscles, keeping the equilibrium in the entire masticatory system is more important than an Angel Class I occlusion that requires displacement of the TMJ to reach the MIC. The biggest advantage of the Dawson classification is that this classification considers the relationship between the MIC and the CR. On the other hand, the $\mathrm{CR}$ considers the position and the status of the TMJs. If the achievement of the MIC requires movement of one or both condyles, the overall result will be a general occlusal imbalance that hampers coordinated muscular activity. Hence, this study used the Dawson classification to investigate the occlusion and its effects and relevance to the TMD. ${ }^{(19,20)}$

The results did not show any significant relationship between TMD and the Dawson and Angel classifications while in some studies, including the studies by Baghaee et $\mathrm{al}^{(2)}$ and Sahebi and Bostani Amlashi (3), occlusal habits have been one of the most effective factors in the development of TMD. The complex issue is that TMJ problems can be responsible for occlusal interactions. There is no specific factor in the occlusion definitively associated with the development of TMJ dysfunction. However, it can be declared that, in some cases, occlusal disorders may be one of the important factors contributing to TMJ dysfunction. More scientific and long-term studies are needed to understand the real relationship between the occlusion and TMD.

According to the results, parafunctional habits (occlusal or cervical wear or both) were not significantly different between subjects with and without TMD. Although, in many other studies, parafunctional habits have had a significant effect on the incidence of TMD. For instance, in the study by Lasemi et al, the frequency of this factor in TMD has been significantly higher. $^{(15)}$ In other studies, a significant correlation has been detected between parafunctional habits and TMD. ${ }^{(14,21,22)}$ The reason for the statistical difference between the aforementioned papers can be due to the lack of patients' knowledge of their parafunctional habits and the absence of wear despite the presence of parafunctional habits.

Although it has not been noted in the literature that parafunctional habits are a decisive factor in TMD development, it has been declared that par- afunctional habits in patients with TMJ problems cause more pain and discomfort. ${ }^{(25-27)}$

Considering the limitations of the present study, including the small group of subjects, it is recommended to examine the prevalence of TMD in an epidemiological study with bigger sample sizes.

\section{Conclusion:}

According to the results of the present study, TMD is common in patients referring to dental clinics with a complaint of pain and discomfort in the temporomandibular region, especially during eating. Among the various factors that have been known to be effective on the incidence of TMD, a history of trauma to the orofacial region has a higher prevalence. Limitations in mouth opening and restrictions on the lateral movements of the jaw are more frequent in TMD patients.

\section{Acknowledgements:}

The authors are grateful to Isfahan Islamic Azad University for supporting and approving of this thesis.

\section{References:}

1. Martin A. Psychotherapy for the MPDS. J Am Dent Assoc. 1974;84(3):924-8.

2. Baghaee B, Ajami B, Hafez B, Khaleseh N, Shirazi AS. Evaluation of the Relationship between Occlusion and Temporomandibular Disorders in Six-Year-Old Preschool Children in Mashhad-Iran. J Mash Dent Sch. 2009;33(4):267-76.

3. Sahebi M, Bostani Amlashi P. Prevalence of temporomandibular disorders and its association with malocclusion in mixed dentition among patients referred to Tehran University Dental School. J Dent Med. 2010;23(3):153-60.

4. Lotfikamran MH, Soltani F, Sohrabi N. Investigating Frequency of Temporo-Mandibular Dysfunctions in Complete Denture Wearers Referring to the Prosthesis Department of Dental School during 2013-2014. JSSU. 2015;23(1):1838-47.

5. Staniszewski K, Lygre H, Bifulco E, Kvinnsland S, Willassen L, Helgeland E, Berge T, Rosén A. Temporomandibular Disorders Related to Stress and HPA-Axis Regulation. Pain Res Manag. 2018;2018:7020751. 
6. Zulqarnain BJ, Khan N, Khattab S. Selfreported symptoms of temporomandibular dysfunction in a female university student population in Saudi Arabia. J Oral Rehabil. 1998 Dec;25(12):946-53.

7. Ebrahimi M, Shahrokhi F, Khorakian F. Orofacial Pain in Doctorate Students (Medical, Dentistry, Pharmacy) in Mashhad University of Medical Sciences in 2012-2013. J Mash Dent Sch. 2015;39(2):99-108.

8. Ebrahimi Saravi M, Khalilian A, Ronaghi H. Prevalence of Temporomandibular Disorders (TMD) and its Signs and Symptoms in Sari Dental School Clinic. J Mazand Univ Med Sci. 2016;26(143):120-8.

9. Schiffman E, Ohrbach R, Truelove E, Look J, Anderson G, Goulet JP, et al. International RDC/ TMD Consortium Network, International Association for Dental Research; Orofacial Pain Special Interest Group, International Association for the Study of Pain. Diagnostic Criteria for Temporomandibular Disorders (DC/TMD) for Clinical and Research Applications: recommendations of the International RDC/TMD Consortium Network and Orofacial Pain Special Interest Group. J Oral Facial Pain Headache. 2014 Winter;28(1):6-27.

10. da Silva CG, Pachêco-Pereira C, Porporatti AL, Savi MG, Peres MA, Flores-Mir C, Canto GD. Prevalence of clinical signs of intra-articular temporomandibular disorders in children and adolescents: A systematic review and meta-analysis. J Am Dent Assoc. 2016;147(1):10-18.e8.

11. Okeson J. Nonsurgical treatment of internal derangements. Oral Maxillofac Surg Clin North Am. 1995;7:63-71.

12.Apostu AM, Checherita L, Pendefunda AC, Ifteni G. The importance of articular mobility testing during TMJ clinical examination technique. Romanian Journal of Oral Rehabilitation. 2018;10(2):144-7.

13.Paulino MR, Moreira VG, Lemos GA, Silva PLPD, Bonan PRF, Batista AUD. Prevalence of signs and symptoms of temporomandibular disorders in college preparatory students: associations with emotional factors, parafunctional habits, and impact on quality of life. Cien Saude Colet. 2018 Jan;23(1):173-86.
14. Sharif M, Aslam A, Rehman A, Hassan SH. Evaluation of ptrygoid muscles in patients with Ttemporomandibular disorders. Pakistan Oral \& Dental Journal. 2017 Sep 30;37(3):492-5.

15. Lee A, He LH, Lyons K, Swain MV. Tooth wear and wear investigations in dentistry. J Oral Rehabil. 2012;39(3):217-25.

16.Kamisaka M, Yatani H, Kuboki T, Matsuka Y, Minakuchi H. Four-year longitudinal course of TMD symptoms in an adult population and the estimation of risk factors in relation to symptoms. J Orofac Pain. 2000 Summer;14(3):224-32.

17.Lasemi E, Navi F, Basir Shabastari S. Prevalence of temporomandibular disorders and it's related factors in dental school of Azad University of Tehran in 2005. J Mash Dent Sch. 2008;32(1):59-64.

18. Baradaran nakhjavani Y, Fardi M. Prevalence of Temporomandibular Disorders in Children age 7-9 years in Primary Schools of Tehran . J Res Dent Sci. 2012; 9 (2) :93-99.

19.Rokaya D, Suttagul K, Joshi S, Bhattarai BP, Shah PK, Dixit S. An epidemiological study on the prevalence of temporomandibular disorder and associated history and problems in Nepalese subjects. J Dent Anesth Pain Med. 2018 Feb;18(1):27-33.

20.Heydari A, Nakhjavani YB, Anaraki EA, Arvan S, Shafizadeh M. Bite Force of 3-6-YearOld Children After Unilateral Extraction of Primary Teeth. J Dent (Tehran). 2018 Jan;15(1):4753.

21.Cordray FE. Three-dimensional analysis of models articulated in the seated condylar position from a deprogrammed asymptomatic population: a prospective study. Part 1. Am J Orthod Dentofac Orthop. 2006 May;129(5):619-30.

22.Dawson PE. A classification system for occlusions that relates maximal intercuspation to the position and condition of the temporomandibular joints. J Prosthet Dent. 1996;75(1):60-6.

23.List T, Wahlund K, Larsson B. Psychosocial functioning and dental factors in adolescents with temporomandibular disorders: a case-control study. J Orofac Pain. 2001 Summer;15(3):218-27.

24. Greene CS. The etiology of temporomandibular disorders: implications for treatment. J Orofac Pain. 2001 Spring;15(2):93-105.

25. Navabi N, Shahravan A, Khoshroo S, As- 
adi L. The Status of Evidence-Based Dentistry in Iran. J Res Dentomaxillofac Sci. 2016; 1 (1) :1-3. 26. Berger M, Szalewski L, Szkutnik J, Ginszt M, Ginszt A. Different association between specific manifestations of bruxism and temporomandibular disorder pain. Neurol Neurochir Pol. 2017 Jan-Feb;51(1):7-11.

27. Motta LJ, Guedes CC, De Santis TO, Fernandes KP, Mesquita-Ferrari RA, Bussadori SK. Association between parafunctional habits and signs and symptoms of temporomandibular dysfunction among adolescents. Oral Health Prev Dent. 2013;11(1):3-7.

Please cite this paper as: Falahati M, Golmohammadi F, Darabi R, jafari M. Evaluation of Temporomandibular Joint Disorders and Related Factors in Patients Referring to Dental School of Isfahan Islamic Azad University in 2019. J Res Dentomaxillofac Sci. 2020;5(3):21-29 